\title{
THERAPIES
}

\section{HEADING:Social pharmacology}

\section{Drug identification by the patient: Perception of patients, physicians and pharmacists}

Drug identification by the patient

Frédéric Tranchard ${ }^{a}$, Julia Gauthier ${ }^{\mathrm{a}}$, Christophe Hein ${ }^{\mathrm{b}}$, Jean Lacombe ${ }^{\mathrm{c}}$, Katharine Brett ${ }^{\mathrm{a}}$, Hélène Villars $^{\mathrm{b}}$, Brigitte Sallerin ${ }^{\mathrm{d}, \mathrm{e}}$, Jean-Louis Montastruc ${ }^{\mathrm{a}, \mathrm{f}}$, Fabien Despas ${ }^{\mathrm{a}, \mathrm{f,g}, \text {,* }}$

${ }^{a}$ Laboratoire de Pharmacologie Médicale et Clinique, Université de Toulouse III Paul Sabatier, 31000 Toulouse, France

${ }^{\mathrm{b}}$ Service de Médecine Gériatrique, Gérontopôle, CHU de Toulouse, 31000 Toulouse, France

${ }^{\mathrm{c}}$ Département Universitaire de Médecine Générale, Faculté de Médecine, Université de Toulouse III Paul Sabatier, 31000 Toulouse

${ }^{\mathrm{d}}$ Service de Pharmacie, CHU de Toulouse, 31000 Toulouse, France

e Laboratoire de Pharmacie Clinique, Faculté de Pharmacie, Université de Toulouse III Paul Sabatier, 31000 Toulouse, France

f Service de Pharmacologie Médicale et Clinique, Faculté de Médecine, Centre de Pharmacovigilance, de Pharmacoépidémiologie et d'Informations sur le Médicament, UMR INSERM 1027, CIC INSERM 1436, Faculté de Médecine, Centre Hospitalier Universitaire, 31000 Toulouse, France

${ }^{\mathrm{g}}$ UMR 1027 Inserm-Université de Toulouse III Paul Sabatier, 31000 Toulouse, France

Received 15 November 2018; accepted 27 January 2019

*Corresponding author. Service de Pharmacologie Médicale et Clinique, CIC 1436, CHU de Toulouse, Toulouse, F-31000, France.

E-mail adress: fabien.despas@univ-tlse3.fr (F. Despas) 


\section{Summary}

Introduction.- Faced with the increasing number of pharmaceutical products on the market, several pharmacovigilance notifications regarding confusion between look-alike and sound-alike drugs have been reported. This study of perception among patients, family physicians and pharmacists aims to evaluate drug identification factors and the risk of errors of confusion for patients.

Material and methods.- Patients were systematically approached in randomly selected pharmacies within the Midi-Pyrénées region in France and invited to complete a questionnaire. Two other questionnaires were respectively sent to family physicians and pharmacists in the same region asking for their opinion on patients' perception of the identification of prescribed medicines.

Results.- Of the 768 patients interviewed, most report identifying their medications by name (brand name: 50\%; generic: $21 \%$ ), while a smaller number cite physical appearance (box: $16 \%$, tablet: $7 \%$ and blister packaging: 3\%). In practice the factors considered most likely to cause confusion by patients relate to drug appearance (look-alike tablets: 28\%, look-alike boxes: $20 \%$ and look-alike blister packaging: 13\%). In contrast, look-alike and sound-alike names (generic and brand names combined) were cited in $31 \%$ of cases. Physicians $(n=345)$ and pharmacists $(n=198)$ understimate that patients identify their treatment by name (physicians: $46 \%$; pharmacists: $26 \%$ vs. patients: $71 \%$ ), reporting instead that problems arise mainly from the appearance of medicines (physicians: identification: $52 \%$ and risk factors for confusion: $74 \%$; pharmacists: identification: $74 \%$ and risk factors for confusion: $83 \%$; versus patients: identification: $26 \%$; risk factors for confusion: $61 \%$ ).

Discussion.- Our study highlights the critical role of medication name in identifying drugs among patients. However, confusion of look-alike tablets or pills figures prominently among fears surrounding medication errors. Despite several notifications of pharmacovigilance, this issue appears to be underestimated within the body of medical literature. Proper identification of medicines by patients is essential to improving medication safety and therapeutic compliance. Concrete measures can be undertaken to reach this goal.

\section{KEYWORDS}

Medication errors; tablets; dosage forms; drug packaging 


\section{Abbreviations}

ANSM: Agence nationale de sécurité du médicament et des produits de santé (French Drug Safety Regulatory Agency)

CNIL : French Data Protection Authority (Commission nationale de l'informatique et des libertés)

ICH: international council for harmonization of technical requirements for pharmaceuticals for human use

INN: international nonproprietary name

URPS: Union régionale des professionnels de santé (Regional Health Professionals Commission) 


\section{Introduction}

In France, most medical consultations result in a medication prescription being given to the patient. Although variability in proportion is observed in European countries [1], patients find themselves at home with prescriptions for drugs with international nonproprietary names (INN) and/or trade names, packaging (boxes and blister packaging) that may be different from one prescription to another and finally the varying tablets or capsules which constitute the dose. The global expansion of the generic drug market has dramatically increased the number of presentations available today. The same principle active substance can therefore now take on different manifestations in terms of packaging or solid oral forms, thus inducing a wide variety in appearance of the medicines. However, the scientific literature is poor on the issue of the drug identification factors used by patients. In addition, the appearance of names in a scientific format and the multitude of packaging and solid oral forms on the market are likely to cause confusion for the patient. Studies have investigated the likelihood of confusion associated with similar names, similarity in appearance of packaging, and in solid oral forms of medications [2-21]. The patient's perception of the risks of confusion has so far been rarely studied in the literature. Moreover, in the journey from clinical examination to the actual taking of the right medication by the patient, it is interesting to note that the physician and pharmacist intervene at different stages and it is therefore wise to evaluate their respective perceptions of the identification factors and risks of confusion of drugs by patients. Indeed, the physician is rarely confronted with the packaging of the drugs and the units of the dose, while the pharmacist is constantly confronted with the packaging and less frequently with the units of the dose. Therefore, the main objective of our study was to evaluate the drug identification factors of a sample of patients from the Midi-Pyrénées region.

The secondary objectives were:

- To identify the risk factors for medication confusion reported by the patients;

- To identify the discriminatory factors of tablets (breakability, inscription, size, shape and color);

- To evaluate subgroup (age, number of different drugs consumed per day, gender and living environment) variations in the methods of identification and factors leading to confusion of drugs; 
- To evaluate the opinion of family physicians and community pharmacists on their perception of patient identification factors and risk factors for confusion of drugs in respective samples from the Midi-Pyrénées region.

\section{Method and materials}

Data collection was done using three evaluation questionnaires; one for patients, one for family physicians and one for community pharmacists. In order to carry out a descriptive analysis between the three subgroups, parts of the questionnaire were common.

\section{Patient protocol}

The patients were recruited from pharmacies in the Midi-Pyrénées region, which were selected by lot. A draw of 12 pharmacies was carried out in January 2015 from the 1088 pharmacies in the Midi-Pyrénées region (Source: "Ordre Régional des Pharmaciens de Midi-Pyrénées," the governing board of Pharmacists in the Midi-Pyrénées region), respecting the population density of each department making up the region. 4 pharmacies in the department of Haute-Garonne, 2 in the department of Tarn and 1 in each of the six other departments (Ariège, Aveyron, Gers, HautesPyrénées, Lot and Tarn-et-Garonne). From a list of 50 randomly preselected pharmacies, the pharmacists responsible for each pharmacy were contacted by telephone in the order that they were selected, to obtain their agreement, allowing the questionnaire to be given to patients in their pharmacy. A total of 8 head pharmacists refused to participate in the study. A unique investigator travelled for one day to each of the selected pharmacies to present the study systematically to all patients upon leaving the pharmacy. Patients were free to choose whether or not to participate in the study by completing the questionnaire they were given. The investigation days took place over the period of $15 / 04 / 2015$ to $15 / 05 / 2015$. After collecting the questionnaires, the exclusion criteria were as follow: being a minor, a patient who declares themselves as non-autonomous in the management 
of their home-based medication, questionnaires containing at least three questions left unanswered or those with answers that were uninterpretable. Questions were defined as uninterpretable if there were multiple responses for a single question or they were illegible. The questionnaire did not include any identifying element for the patient, thus respecting anonymity. The French Data Protection Authority (Commission nationale de l'informatique et des libertés [CNIL]) confirmed that it was not necessary to file a specific declaration.

\section{Physician protocol}

After validation of the study protocol by the Regional Health Professionals Commission (Union régionale des professionnels de santé [URPS]) of the Midi-Pyrénées region, the questionnaire for general practitioners was written and configured in LimeSurvey ${ }^{\circledR}$ format allowing the completion of the questionnaire online. The link to access the Internet portal was sent by email on 10/03/2015 to the entire mailing list of general practitioners belonging to the URPS Midi-Pyrénées network, i.e. 2351 recipients. A reminder, again by email, was sent to the entire mailing list on 20/03/2015, this time with 2345 recipients. The terms of anonymity of the answers were respected. Only questionnaires completed in full by physicians were included in the analysis. Data collection was done at URPS Midi-Pyrénées on 23/04/2015.

\section{Pharmacist protocol}

The questionnaire was printed and sent to the 1037 pharmacies of the Midi-Pyrénées region (source: National Association of Pharmacists) in February 2015 through the wholesaler-distributor network. The questionnaire was also sent to the governing board of Pharmacists in the Midi-Pyrénées region, who then went on to send the questionnaire by e-mail to all pharmacies in the region. Pharmacists who responded to the questionnaire were given the opportunity to return by post, e-mail or fax. All questionnaires were collected during the month of April 2015. The exclusion criteria for the 
analysis were: questionnaires containing at least three questions left unanswered or whose answers were considered uninterpretable (multiple or illegible answers.).

\section{Statistical analysis}

A descriptive analysis of the studied parameters was carried out. Results were expressed as mean \pm standard deviation and/or median, minimum and maximum for quantitative variable and as percentages for categorical variables. A Student T-test was performed for the comparison of the variables according to groups of interest. A subgroup analysis compared patients aged 75 years and older $(\mathrm{n}=91)$ to the rest of the sample, among other subgroup analyses. Analyses were performed with Graphpad ${ }^{\circledR}$ software version 5.0.

\section{Results}

A total of 807 patient questionnaires, 389 physician questionnaires and 210 pharmacist questionnaires were collected. After application of the exclusion criteria, 768 patient questionnaires, 345 physician questionnaires and 198 pharmacist questionnaires were included in the analysis. The characteristics of the patients, physicians and pharmacists included are reported in Table 1 . The patients and pharmacists who responded to the survey were mostly women, while the physicians were mostly men. The most represented age class among patients was between 40 and 54 years, the average age of the physicians was $49.7 \pm 11.0$ years and the average age of the pharmacists was 45.3 \pm 5.7 years. The environment was mainly rural for the patients and pharmacists and semi-urban for the physicians. Patients reported taking on average $1.89 \pm 2.22$ medications per day. A total of $10 \%$ of the sample reported having already confused two tablets or capsules $(8.6 \%$ among patients taking 0-1 drugs daily, vs $11.8 \%$ among patient taking at least 2 drugs daily, difference not statistically significant). Figure 1a shows the drug identification factors for patients. The majority of patients reported identifying their medications by trade name $(50 \%)$ or generic name $(21 \%)$, the 
appearance of the box was reported in $16 \%$ of cases. Physicians reported their perception being for $42 \%$ that patients identify their medications using the trade name, 30\% through the appearance of the tablet and $19 \%$ the appearance of the box. $40 \%$ of pharmacists reported the appearance of the tablets, $33 \%$ the appearance of the box and $24 \%$ the trade name. Regarding the factors of confusion reported by patients (Figure 1b), similar names (trade names and generic names together) were most commonly reported (31\%) followed by similar tablets (28\%) and similar boxes (20\%). The physicians estimated in $48 \%$ of respondents that it is the similar tablets; the similar names (22\%) and similar boxes $(21 \%)$ that is at risk of confusion by the patients. Pharmacists on the other hand, reported similar tablets (49\%), similar boxes $(32 \%)$ and similar names $(15 \%)$. In terms of the method of identification of tablets or capsules once they have been removed from their packaging (Figure 1c), 26\% of responding patients reported identifying them from the engraved or printed inscription on them, $25 \%$ said they refer to their color and then $22 \%$ to the geometric shape. The majority of physicians reported that patients identify from the color (56\%), followed by the inscription (19\%), then their shape (15\%). Pharmacists believed that patients identify mainly by color $(58 \%)$, followed by shape (21\%), then the inscription (16\%).

Subgroup analyses of the included patients were carried out. An analysis comparing patients aged 75 years and older $(n=91)$ compared to the remainder of the sample $(n=676)$ showed that the older patients took on average $4.05 \pm 2.55$ different medications per day versus $1.61 \pm 2.02$ for the younger patients $(\mathrm{P}<0.0001)$. In terms of drug identification factors, the older patients reported identifying less frequently from the trade name (32\%) compared to younger patients $(52 \%$ in the younger population; $\mathrm{p}=0.003$ ). In addition, older patients reported in $15 \%$ of cases to identify drugs from the appearance of the tablet, whereas younger patients reported this in only $6 \%$ of cases $(\mathrm{P}=0.0045)$. Regarding factors of confusion of medications, patients aged 75 years and over reported in $17 \%$ of cases that similar names were a risk of confusion while this was reported in $33 \%$ of cases among younger patients $(\mathrm{P}=0.0025)$. Patients aged 75 years and over reported in $13 \%$ of cases that engraved or printed inscriptions on the tablet or capsule were useful in identifying a drug, while younger patients reported this factor in $28 \%$ of cases $(\mathrm{P}=0.0037)$.

Subgroup analysis comparing patients taking 5 or more drugs daily $(\mathrm{n}=98)$ compared to the rest of the patients in the sample $(n=670)$ shows that the tablet's appearance for identifying drugs was reported in $15 \%$ of patients defined as polymedicated versus $6 \%$ of patients taking less than 5 drugs per day $(\mathrm{p}=0.0005)$. Concerning the factors of confusion, it was found that, similar tablets were reported in $34 \%$ of patients defined as polymedicated versus $25 \%$ in patients taking less than 5 drugs per day $(\mathrm{P}=0.0365)$, similar names was reported in $21 \%$ of patients defined as 
polymedicated versus $33 \%$ in patients taking less than 5 medications per day $(\mathrm{P}=0.0195)$. Regarding the identification of tablets or capsules, the color was reported in $35 \%$ of patients defined as polymedicated versus $24 \%$ of patients taking less than 5 drugs per day ( $p=0.0133$ ), engraved or printed inscription was reported in $15 \%$ of patients defined as polymedicated versus $27 \%$ of patients taking less than 5 drugs per day $(\mathrm{p}=0.0176)$. Finally, with regards to the subgroup analysis of gender, it appears that for women $(n=507)$, the appearance of the box was more frequently reported as a drug identification factor, $18 \%$ versus $11 \%$ in men $(\mathrm{p}=0.0146)$.

\section{Discussion}

Our results show that the majority of patients say they identify drugs by their names (commercial name: 50\% and generic name: 21\%). The appearance of the drug (box appearance: 16\%, tablet appearance: $7 \%$ and blister packaging appearance: $3 \%$ ) is less frequently reported. However, the factors considered at risk of confusion by the patients are mainly related to the appearance of the drugs (similar tablets: $28 \%$, similar boxes: $20 \%$ and similar blister packaging: $13 \%$ ) whereas the similarity of the commercial and generic names comprises only $31 \%$ of cases. The opinion of doctors and even more pharmacists on the perception of patients is mainly focused on the appearance of drugs (cumulative results for the appearance of tablets, boxes and blister packaging):

- Patients: identification: $26 \%$ and confusion factors: $61 \%$;

- Physicians: identification: $52 \%$ and confusion factors: $74 \%$;

- Pharmacists: identification: $74 \%$ and confusion factors: $83 \%$;

It is not surprising that patients report identifying their medications primarily on the basis of the name, since it is the generic or commercial name that most precisely defines the drug taken. This is also what is written on the doctor's prescription and to which the patient refers in order to take their treatment. Recognition of the box (secondary packaging) also plays an important role in drug identification. An abundance of medical literature on the issue of name confusion or similar packaging [4-16] supports this assertion. 
On the other hand, it is interesting to note that when patients are asked to target a cause of error when drugs are mixed up, confusion of the tablets immediately follows the confusion of names (commercial or generic combined) and thus precedes the confusion of boxes and blister packaging. Physicians and pharmacists believe that it is the similarities among solid oral forms that is most at risk of errors of confusion for patients.

Our study confirms the greatest consumption of medication by elderly patients as compared to younger patients. An average of 4.05 different drugs taken each day among patients with an age of 75 years or older is consistent with data from the body of French medical literature [22-24], although a more recent study reported higher drug consumption in a French population over 65 [25].

For subgroup analyses, the relative importance of the brand name in drug identification is lower in patients 75 years or older compared to younger patients, while the appearance of the tablet appears to be more important in this older population compared to younger patients. The similarity of names is also less cited as a risk factor for medication confusion in patients 75 years of age or older compared to younger patients. Similarly, compared to younger patients, older patients seem to pay less attention to the possible markings on tablets in order to differentiate them.

Patients taking at least five different medications a day place relatively more importance on tablet appearance than patients taking fewer medications in order to identify them. Polymedicated patients are comparatively more afraid of tablet similarity as a confounding factor for drugs compared to patients taking fewer than five different distinct medications per day, whereas they are less fearful of similarities in name. Polymedicated patients are also more sensitive to color to differentiate tablets and place less importance on pill markings compared to patients who consume fewer drugs. In addition, there are more errors by confusion in patients taking at least five different drugs per day compared to patients taking less.

Compared to men, women are more sensitive to the appearance of the box to identify drugs. The analysis according to the living environment (urban, semi-urban and rural) did not show statistically significant differences on all the variables studied.

According to our study, effective identification of drugs by patients, in the sense of taking the medications safely, thus prominently involves visually recognizing the tablets, particularly in elderly patients. Indeed, the oral administration of the drug is the final step in the process of taking it, which follows prescription, delivery and preparation. It is therefore the last step to identify and 
thus avoid a possible medication error occurred upstream [26]. Visual recognition of the tablet or capsule for identification purposes is therefore essential for the prevention of medication errors. On the other hand, the confusion of names and similar packaging is a very real problem for health professionals, from the prescribing doctor to the pharmacist who delivers the medicines, to the nurse who prepares pillboxes at the patients' home or at the hospital [27].

According to patients, color and the presence of an inscription appear to be the predominant organoleptic characteristics in the identification of tablets or capsules. Doctors and pharmacists, for their part, cite color as the key to identifying medicines once they are out of their packaging. The introduction of a staining of the solid oral forms according to the pharmacological class of the active substance that they contain according to the anatomical therapeutic chemical classification system would simplify the identification of drugs as well as a facilitation of the analysis of patient pillboxes for the purpose of preventing errors. However, color-coding seems to be debated while Tall Man lettering or the addition of the drug indication on the prescriptions and labels appear as interesting means to differentiate medications [28-30]. It may also be useful to adopt an international regulation governing the marking of solid oral forms by engraved or printed inscriptions, assigning a mandatory code specific to each pill on the market, in order to ensure standardization of the drug identification. Indeed, some inscriptions are sometimes acronyms (for example: VGR $=$ Viagra $^{\circledR}$ ) but there is no international standardization. If supported and motivated by the International Council for Harmonization of Technical Requirements for Pharmaceuticals for Human Use (ICH), such standardization would reduce the number of similar solid oral forms on the global market, minimizing the risk of drug error at the time of preparation and administration of tablets or capsules, both of which have been identified by physicians as the most error-prone, as also confirmed by the statistics collected in the activity report on medication errors published in 2010 by the French Drug Safety Regulatory Agency (Agence nationale de sécurité du médicament et des produits de santé [ANSM]) [6].

Of the 11,337 tablets present within the French pharmaceutical market in August 2015, 2,266 were both round, white and measured between 8 and $12 \mathrm{~mm}$ in diameter, or 1 in 5 [31], illustrating a clear lack of diversity of appearance of tablets and therefore a potential risk for medication confusion.

However, our study has also limitations. The sex ratio in favor of women among the included patients could be explained by more frequent visits by women to the pharmacy. The protocol was to offer the questionnaire to all subjects leaving the pharmacy, regardless of reason for 
purchase. The parapharmacy clientele, with a female majority, was therefore also assessed. Some of the questions in the Patient Questionnaire and the Physician Questionnaire relied on the memory of respondents, especially regarding possible past medication confusion, thus introducing memory bias. The question about tablet and capsule identification frequently led to multiple responses leading to censoring this question more often than others at the time of the collection of data (whereas a single box should be checked to define the answer as interpretable). After completing the questionnaire, many patients expressed difficulty in deciding whether or not checking only one box, resulting in multiple responses. In order not to have a too long questionnaire, we had to limit the number of questions. Thus some error factors were not addressed in the questionnaires, such as the recent change of treatment, the dispensing of generic drugs from another laboratory, medication management at home (use of a pillbox, separate or mixed stocks of medications between spouses...). Finally, our study focused only on prescription drugs. An underestimation of the problem of confusion of medicines can result, because in the home of the patients can also be drugs not subject to medical prescription, food supplements or other substances appearing similar to prescribed medications [32-33]. Moreover, this problem of confusion of similar drugs is inevitably underestimated because of the obvious underreporting of adverse drug reactions and medication errors [34-35]. Improving the prevention of medication errors and adverse drug reactions is inexpensive with regard to the health savings it is likely to achieve if it is executed effectively. It remains however dependent on the proper notification of adverse events to the regulatory agencies. We must therefore strive to continue raising awareness on this topic with health professionals and patients. In a pragmatic approach, it seems important to keep encouraging patients to bring their unused drugs back to the pharmacy in order to limit stocks at home and thus reduce the risk of drug confusion. Although it suffers from weaknesses inherent to its design, it is to our knowledge the first study looking at drug identification methods and taking into account both the opinion of patients but also that of prescribing doctors and dispensing pharmacists.

Just as physician software includes a function to report to the prescriber potential drug interactions in the form of alerts when prescribing a medication, it is technically feasible to develop a similar module to preemptively alert pharmacists about the risk of confusion when dispensing similar tablets or capsules. An alert in the form of a "pop-up" showing photographs of the tablets or capsules in question would then appear on the screen. If the pharmacist did not have the possibility to replace one of the tablets with another of the same principle active substance at the same dosage, they could nevertheless turn the computer screen toward the patient to warn them against the risk of confusion with photographic support. This module would provide text descriptions regarding color, 
shape, dimensions, inscription and scoring, as well as photographs of solid oral forms. Based on perception studies, we could define the standardized similarity criteria in order to determine the sensibility and specificity of the messages in order to limit the phenomenon of "alert fatigue" (decrease in attentiveness to the alert following excessive repetition) [36]. In addition to its daily usefulness at pharmacies, this tool could lead to the implementation of software for prescribing physicians so that they can know in a few clicks if a clinical or pathological state could be attributed to confusion of similar tablets or capsules, depending on the drugs dispensed by the pharmacist. Similarity of tablets or capsules must be fully considered as a drug interaction.

\section{Acknowledgement}

John A. Ice, for English translation.

\section{Funding}

This research did not receive any specific grant from any funding agency in the public, commercial, or not-for-profit sector.

\section{Disclosure of interest}

The authors declare that they have no competing interest. 


\section{References}

[1] Direction de la recherche, de l'évaluation, des études et des statistiques. Etudes et résultats. Les prescriptions des médecins généralistes et leurs determinants. vol 440. November 2005. http://drees.solidarites-sante.gouv.fr/IMG/pdf/er440.pdf [Accessed 22 March 2019 (12 pp.)].

[2] Mills GA. Look-alike threats to unit dose safety. Hosp Pharm1985;20:168-170.

[3] Mira JJ, Orozco-Beltrán D, Pérez-Jover V, Martínez-Jimeno L, Gil-Guillén VF, CarratalaMunuera $\mathrm{C}$, et al. Physician patient communication failure facilitates medication errors in older polymedicated patients with multiple comorbidities. Fam Pract 2013;30:56-63.

[4] World Health Organization. Patient safety solutions. Look-alike, sound-alike medication names. 2007. http://www.who.int/patientsafety/solutions/patientsafety/PS-Solution1.pdf [Accessed 22 March 2019 (4 pp.)]

[5] Lefebvre L. Erreurs de médication: ressemblances et différences. Centre de toxicologie clinique, Institut National de Santé Publique du Québec. 1997. https://www.inspq.qc.ca/toxicologieclinique/erreurs-de-medication-ressemblances-et-differences [Accessed 22 March 2019].

[6] Agence nationale de sécurité du médicament et des produits de santé. Bilan d'activité de l'année 2009. Guichet erreurs médicamenteuses. 2010. http://ansm.sante.fr/var/ansm_site/storage/original/application/7881b7874c6cf3eb29e3a6657c84fa7 0.pdf [Accessed 22 March 2019 (28 pp.)].

[7] Lambert BL, Lin SJ, Tan H. Designing safe drug names. Drug Saf 2005;28:495-512.

[8] Gabriele S. The role of typography in differentiating look-alike/sound-alike drug names. Healthc Q Tor Ont 2006;9 Spec No:88-95.

[9] Lavon O, Ben-Zeev A, Bentur Y. Medication errors outside healthcare facilities: A national poison centre perspective. Basic Clin Pharmacol Toxicol 2014;114:288-92.

[10] Schnoor J, Rogalski C, Frontini R, Engelmann N, Heyde CE. Case report of a medication error by look-alike packaging: a classic surrogate marker of an unsafe system. Patient Saf Surg 2015 Mar 13;9:12. 
[11] Thielmeier KA. Drug packaging similarities require extraordinary vigilance. J Clin Anesth 1996;8:688-9.

[12] Pathak A, Senard JM, Bujaud T, Bagheri H, Lapeyre-Mestre M, Tressieres MC, et al. Medication error caused by confusing drug blisters. Lancet 2004;363:2142.

[13] Prabhakar A, Malapero RJ, Gabriel RA, Kaye AD, Elhassan AO, Nelson ER, et al. Medication errors in anesthesia. J Med Pract Manag MPM 2015;30(6 Spec No):41-3.

[14] Shah HD, Shah M. A case of look-alike medication errors. Indian J Pharmacol 2011;43:4823.

[15] Ogboli-Nwasor E. Medication errors in anaesthetic practice: a report of two cases and review of the literature. Afr Health Sci 2013;13:845-9.

[16] Ioannides KLH, Kassutto Z. Case of accidental overdose due to look-alike medication bottles. Visual Journal of Emergency Medicine 2017;8:27-8. 10.1016/j.visj.2017.03.007.

[17] Langford N, Graham-Clarke E. Confusing tablets. Lancet 2005;366:154.

[18] Prakash B. Sad confusion of look-alike tablets. Indian Dermatol Online J 2010;1:50.

[19] Walliser G, Grossberg R, Reed MD. Look-alike medications: A formula for possible morbidity and mortality in the long-term care facility. J Am Med Dir Assoc 2007;8:541-2.

[20] Tranchard F, Hein C, Lacombe J, Villars H, Montastruc JL, Despas F. Medication errors and look-alike tablets: the splitting issue. Pharmacoepidemiol Drug Saf 2016 Sep;25(9):1002-3.

[21] Jacob T, Garrick R, Goldberg MD. Recurrent lactic acidosis and hypoglycemia with inadvertent metformin use: a case of look-alike pills. Endocrinol Diabetes Metab Case Rep 2018 Jan 5;2018:pii: 17-0148.

[22] Legrain S. Consommation médicamenteuse chez le sujet âgé. Haute autorité de santé. 2005. http://www.has-

sante.fr/portail/upload/docs/application/pdf/pmsa_synth_biblio_2006_08_28_16_44_51_580.pdf [Accessed 22 March 2019 (16 pp.)]

[23] Auvray L, Sermet C. Consommations et prescriptions pharmaceutiques chez les personnes âgées : un état des lieux. Gérontologie et société 2002;25(103):13-27. 
[24] Huon JF, Lenain E, LeGuen J, Chatellier G, Sabatier B, Saint-Jean O. How drug use by french elderly patients has changed during the last decade. Drugs Real World Outcomes 2015;2(4):327-33.

[25] Rhalimi M, Rauss A, Housieaux E. Drug-related problems identified during geriatric medication review in the community pharmacy. Int J Clin Pharm 2018 Feb;40(1):109-18.

[26] Sattar SP, Gastfriend DR. Visual inspection of medications in preventing unexplained relapses. Ann Pharmacother 2002;36:1648-9.

[27] James KL, Barlow D, McArtney R, Hiom S, Roberts D, Whittlesea C. Incidence, type and causes of dispensing errors: A review of the literature. Int J Pharm Pract 2009;17:9-30.

[28] Larmené-Beld KHM, Alting EK, Taxis K. A systematic literature review on strategies to avoid look-alike errors of labels. Eur J Clin Pharmacol. 2018 Aug;74(8):985-9=93.

[29] Ciociano N, Bagnasco L. Look alike/sound alike drugs: A literature review on causes and solutions. Int J Clin Pharm.= 2014 Apr;36(2):233-42.

[30] Seoane-Vazquez E, Rodriguez-Monguio R, Alqahtani S, Schiff G. Exploring the potential for using drug indications to prevent look-alike and sound-alike drug errors. Expert Opin Drug Saf.= 2017 Oct;16(10):1103-9.

[31] Tranchard F. Discrimination des médicaments et similarité d'apparence des formes orales solides ; perception des patients et des médecins. Thèse d'exercice, Université Toulouse III Paul Sabatier, 2015. http://thesesante.ups-tlse.fr/969/1/2015TOU31072.pdf [Accessed 22 March 2019 (62 pp.].

[32] Garriott JC, Simmons LM, Poklis A, Mackell MA. Five cases of fatal overdose from caffeine-containing « look-alike » drugs. J Anal Toxicol 1985;9:141-3.

[33] Mueller SM. Neurologic complications of phenylpropanolamine use. Neurology 1983;33:650.

[34] Hazell L, Shakir SAW. Under-reporting of adverse drug reactions : a systematic review. Drug Saf 2006;29:385-96. 
[35] Académie Nationale de Médecine. Signalement des évènements indésirables en médecine Protection juridique. 2012. http://www.cclin-arlin.fr/nosopdf/doc12/0033522.pdf [Accessed 22 March 2019 (7 pp.)].

[36] Van der Sijs H, Aarts J, Vulto A, Berg M. Overriding of drug safety alerts in computerized physician order entry. J Am Med Inform Assoc 2006;13:138-47. 
$26 *$ Corresponding author: florence.mouchet@ensat.fr

\section{microcosms} Gauthier $^{1}$ cedex 9, France

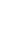
(1)

(2)

Lauris Evariste ${ }^{1}$, Antoine Mottier ${ }^{1}$, Laura Lagier ${ }^{1}$, Stéphanie Cadarsi $^{1}$, Maialen Barret ${ }^{1}$, Cyril Sarrieu $^{2}$, Brigitte Soula ${ }^{2}$, Florence Mouchet $^{1 *}$, Emmanuel Flahaut ${ }^{2}$, Eric Pinelli ${ }^{1}$ and Laury

${ }^{1}$ EcoLab, Université de Toulouse, CNRS, INPT, UPS, Toulouse, France

${ }^{2}$ CIRIMAT, Université de Toulouse, CNRS, INPT, UPS, UMR CNRS-UPS-INP N5085, Université Toulouse 3 Paul Sabatier, Bât. CIRIMAT, 118 route de Narbonne, 31062 Toulouse 
Extensive development of new applications using graphene based materials such as graphene oxide (GO) increases its potential release and occurrence into aquatic environments, raising the question of its biological and ecological risks. As standardized single-species-based assays fail to highlight toxicological pathways implying interactions between organisms, the use of micro/mesocosms appears as a good solution to fill the lack of environmental realism inherent to these tests. In this work, experiments were achieved using microcosm systems to expose a reconstituted food chain to $\mathrm{GO}$ at environmentally-relevant concentrations $\left(0.05\right.$ and $0.1 \mathrm{mg} . \mathrm{L}^{-}$ ${ }^{1}$ ). The trophic chain was composed of a consortium of algae and bacteria as primary producers, chironomid larvae as primary consumers and decomposers while larvae of the amphibian Pleurodeles waltii constituted the secondary consumers. Monitoring of multiple ecotoxicological and ecological endpoints allowed to observe changes in bacterial communities while no toxic effects were noticed in chironomids. However, chironomids feeding behaviour changed as a consequence of GO contamination, leading to an increase in leaf litter consumption. Genotoxic effects were noticed in Pleurodeles larvae. This study highlights the importance of using such experimental systems to better encompass the ecotoxic potential of GO through the determination of toxicological routes and consequences on ecosystem's functioning. 
Carbon-based nanoparticles (CBNs) possess unique properties (high surface area, electrical and thermal conductivity, mechanical strength and optical transmittance) triggering tremendous scientific expectations for development of a wide range of industrial applications [1-5]. Graphene oxide (GO) is a chemically oxidized form of graphene that consists of a single atom thick two-dimensional sheet of carbon atoms containing epoxide, hydroxyl and carboxyl groups. Due to its high oxygen content, GO is a hydrophilic nanomaterial that is stable when dispersed in aqueous media [6]. As other engineered nanoparticles (ENPs), GO is likely to be released into the ecosystems at any stage of its life cycle, from the production, the uses and the waste treatments of the substance and may cause environmental and health issues [7-10]. Due to its hydrophilic properties associated to high surface area and chemical functions, GO could potentially react with many components of the environment, modifying its ecological risk toward aquatic ecosystems [11].

Numerous toxicological studies of graphene-based nanomaterials (GBNs) were performed on mammalian cell lines or human-related biological models [8,12-14] while potential effects on environmentally relevant models were not sufficiently investigated [15,16]. In addition, existing studies used assays performed through exposure of single species to CBNs are essential to understand toxicological mechanisms, these tests are poorly representative of environmental conditions [17]. For this purpose, the use of more complex systems such as micro-mesocosms was shown to be efficient to assess the ecotoxic potential of multiple contaminants under environmentally relevant conditions [17-20]. These experimental systems allow measurement of both ecosystemic and toxicological endpoints in multiple interacting species or communities, from different trophic levels, after direct exposure of organisms as well as through trophic routes [21-23]. Thus, performing this type of study is becoming crucial for a better understanding of potential environmental issues implying any contaminant and including GO.

Previous works mainly focused on the ecotoxicological effects of GO on organisms from lower trophic levels such as bacteria or algae exposed in single culture. Antibacterial activity of GO was identified [24-26] and among multiple GBNs, GO was shown to exert the highest cytotoxicity toward the bacterial model Escherichia coli [27]. Concerning adverse effects toward algae, it was demonstrated that GO exposure led to Chlorella sp. growth inhibition and produced membrane damages through mechanical actions as well as oxidative stress induction [28,29]. Studies carried out on organisms from higher trophic levels (i.e. primary and secondary consumers) are still scarce. It was indicated that GO induced behavioral disturbances of 
crustacean larvae (Amphibalanus amphitrite and Artemia salina) and was strongly accumulated in digestive tract [30,31]. In amphibians, similar accumulation of GO was observed in gut of Xenopus laevis tadpoles and growth was inhibited at high concentrations [32]. Observation of toxicological effects induced by GO on low trophic level organisms raises the question of possible larger scale consequences through trophic network and on ecosystem functioning.

The aim of this study was to investigate the toxicity of GO under realistic conditions to go further in the understanding of its impact on organisms occupying multiple trophic levels and to determine potential consequences on ecosystem's functioning. For this purpose, microcosm systems were used to expose organisms interacting through a reconstituted trophic chain. The biological models retained for the study were chosen for being key species representative of a simplified trophic chain from pond ecosystems [33,34]. Although they have significant ecological functions, number of human activities are threatening these ecosystems as well as the biodiversity they host, especially endangering species such as amphibians [35]. For this purpose, the benthic diatom Nitzschia palea and a bacterial consortium were settled to form a biofilm providing food for chironomid larvae (Chironomus riparius) as primary consumers and decomposers. Secondary consumers were constituted by the carnivorous larvae of the amphibian, Pleurodeles waltl, the Spanish newt, that feed on macro-invertebrates. As microbial communities are at the very basis of every ecosystem functioning and due to their implication on global nutrient cycling [36], changes in microorganism community composition were monitored during the experiment. In terms of toxicological endpoints, survival and growth parameters of the consumers were controlled as well as morphological deformities in chironomids and count of micronucleated erythrocytes in Pleurodeles [37,38]. Analysis of chironomids mouthpart deformities constitutes a marker of teratogenesis that is widely used in pollution monitoring as well as micronuclei induction that is a good indicator of genotoxicity, integrating aneugenic and clastogenic effects that is used as endpoint in ISO 21427-1 procedure. Both teratogenic and genotoxic biomarkers are considered as highly predictable ecological endpoints at the population, community and ecosystem level [39]. The process of leaf litter decomposition ensured by chironomids and bacteria was also investigated, as it constitutes an interesting functional ecological marker integrative of lower trophic level activities [22,40].

\section{Material and Method}

\subsection{Graphene oxide nanoparticles:}

Graphene oxide nanoparticles were produced from twisted ribbon-shaped carbon nanofibers (GANF®) processed by Hummer method [41] and supplied by Antolin Group. Full 
characterization of the tested material was already presented in earlier work [42] and data are summarized in table 1. Both experiments were performed within few weeks. GO was stored as dry powder in the dark and dispersions were prepared extemporaneously in order to avoid any possible change of material characteristics.

Table 1: Physico-chemical characteristics of GO used for the experiment. at. \%: atomic \%; Csp2: sp² carbon; Sat.: shake-up satellites ( $\pi$ to $\pi^{*}$ transitions); TEM: transmission electron microscope; HRTEM: high resolution TEM; BET: Brunauer-Emett-Teller.

\begin{tabular}{cc}
\hline & Graphene Oxide \\
\hline Carbon content & 69.0 at. $\%$ \\
Oxygen content & 31.0 at. $\%$ \\
Csp2 graphene & 35.5 at. $\%$ \\
C-OH/C-O-C & 24.7 at. $\%$ \\
C=O & 2.5 at. $\%$ \\
O=C-O & 5.3 at. $\%$ \\
Sat. & 1.4 at. $\%$ \\
Number of layers (HRTEM) & $1-5$ \\
Lateral size (TEM) & 0.2 to $8 \mu \mathrm{m}$ \\
Specific surface area (BET) & $228 \pm 7 \mathrm{~m}^{2} . \mathrm{g}^{-1}$ \\
\hline
\end{tabular}

\subsection{Stability of graphene oxide suspension in exposure media}

The stability of the suspension of GO was evaluated using a Turbiscan LAB (Formulation) equipment, at room temperature. Suspensions were prepared at $10 \mathrm{mg} . \mathrm{L}^{-1}$ in deionized water (EDI) or Volvic water immediately before analysis. This concentration was assessed as low GO concentrations low could not be detected by TurbiscanTM. The suspension was bath sonicated for 2 minutes before insertion into the measurement chamber. Sedimentation was monitored for $24 \mathrm{~h}$ with 1 scan per minute by measuring during each scan the transmission of the suspension vs the height in the vial. In case of sedimentation, it is thus expected that the transmission will increase at the heights where particles have settled down, while it should decrease where sedimented particles are present.

\subsection{Microcosm exposure}

Nine microcosms (glass tanks 60x30x30 cm, L x W x H) were filled with 6780 grams of reconstituted sediment: (96.6\% of silica sand, $2.4 \%$ of kaolinite and $1 \%$ of $\mathrm{CaCO}_{3}$ ) and 40 liters of the commercial natural spring water Volvic $®\left(\mathrm{Ca}^{2+}: 12 \mathrm{mg} \cdot \mathrm{L}^{-1}, \mathrm{Na}^{+}: 12 \mathrm{mg} . \mathrm{L}^{-1} \mathrm{Mg}^{2+}\right.$ : $8 \mathrm{mg} . \mathrm{L}^{-1}, \mathrm{~K}^{+}: 6 \mathrm{mg} . \mathrm{L}^{-1}, \mathrm{Cl}^{-}: 1 \mathrm{mg} . \mathrm{L}^{-1}$ and $\left.\mathrm{Si}: 32 \mathrm{mg} . \mathrm{L}^{-1}\right)$ as used in previous microcosm studies [21,43-45]. In order to maintain correct oxygenation, each tank was equipped with a 
recirculation system. Light was provided by fluorescent tubes (JBL solar ultra, Natur $9000^{\circ} \mathrm{K}$ LT 24 WT5-HQ) and photoperiod was set on 12:12 light/night. Temperature $\left(21.5 \pm 0.9^{\circ} \mathrm{C}\right)$, conductivity $\left(234.3 \pm 9.1 \mu \mathrm{S} . \mathrm{cm}^{-1}\right), \mathrm{pH}(7.9 \pm 0.4)$, redox potential $(306.6 \pm 17.3 \mathrm{mV})$ and dissolved oxygen $\left(9.1 \pm 1.2 \mathrm{mg} . \mathrm{L}^{-1}\right.$ ) were monitored continuously (Ponsel Odeon open X probes kit). Nitrogen products $\left(\mathrm{NO}_{3}{ }^{-}, \mathrm{NO}_{2}{ }^{-}\right.$and $\left.\mathrm{NH}_{4}{ }^{+}\right)$were monitored twice a week using a HI83203 photometer (Hanna instruments) and dissolved organic carbon was measured at each sampling time by infrared detection of $\mathrm{CO}_{2}$ produced by catalytic oxidation at $680^{\circ} \mathrm{C}$ (Table S1).

Three different exposure conditions were tested: control (natural spring water), graphene oxide at the final concentration of $0.05 \mathrm{mg} \cdot \mathrm{L}^{-1}$ and graphene oxide at the final concentration of $0.1 \mathrm{mg} . \mathrm{L}^{-1}$. Each condition was tested in triplicate with random assignation of the microcosms. Contamination of the microcosms by graphene oxide nanoparticles was performed sequentially through the addition of $1 / 12^{\mathrm{e}}$ of the final concentration over 10 days to reach the final concentration indicated by the condition name $\left(0.05 \mathrm{mg} . \mathrm{L}^{-1}\right.$ and $\left.0.1 \mathrm{mg} . \mathrm{L}^{-1}\right)$ (Figure 1$)$.

The progress of the experiment and the order in which the different organisms were introduced are shown in Figure 1. Senescent alder leaves (Alnus glutinosa Gaertn.) (3.2 g dry mass/mesocosm) were added the first week for measurement of decomposition rate and microorganisms (microbial consortium $1 \times 10^{6}$ cells $/ \mathrm{ml}$ and Nitzschia palea $2.05 \times 10^{4}$ cells $/ \mathrm{ml}$ ) were allowed to settle and develop during 3 weeks without any contamination. This duration was necessary for the development of the primary producers leading to the formation of a biofilm covering the whole sediment [44] and equilibration of nitrogen products in the microcosms by bacteria. The first samplings of the biofilm and water were performed to analyze microorganism communities prior to contamination and addition of primary and secondary consumers (T0). Other organisms, bred in the laboratory facilities, were added sequentially to the microcosms (Fig. 1). 800 Chironomus riparius individuals aged of $72 \mathrm{~h}$ and 15 Pleurodeles waltii individuals at stage 53 of development [46] were added during the fourth week to allow an exposure duration of 13 and 10 days respectively. These durations were chosen in order to ensure that the surviving $C$. riparius would develop until the stage 4 (required condition for teratogenicity analysis). Moreover, chironomids were added prior to the newts to grow and start to accumulate GO in order to ensure trophic transfer towards the secondary consumers. For Pleurodeles, exposure duration of 10 days is necessary for genotoxic assessment at low dose [47]. A second sampling of the microorganisms was performed when half of the final GO dose was reached (T1) and at the end of the exposure when the full dose was reached (T2). Xenopus laevis $(\mathrm{n}=40)$ at stage 50 of development [48] were added during the week 5 as a food 
complement for Pleurodeles larvae. Chironomids, Pleurodeles and alder leaves were also sampled at $\mathrm{T} 2$, constituting the end of the experiment.

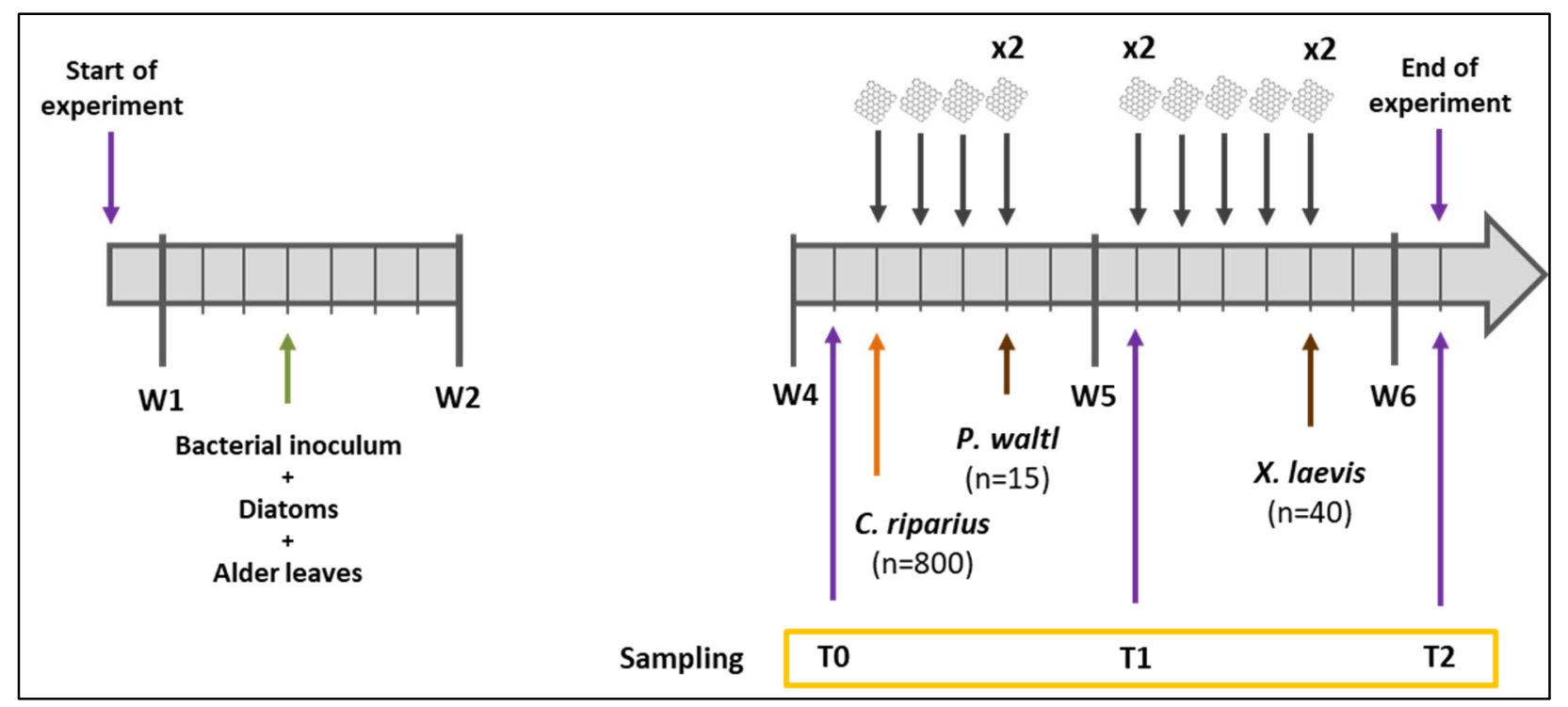

Figure 1: Scheme of the time-progress of the microcosm experiment. Bacterial consortium and diatoms were allowed to settle and develop to colonize the sediment and alder leaves during 3 weeks. Prior to the addition of other organisms and GO, a first sampling of the primary compartment was performed (T0). GO was then added sequentially in the microcosms over 2 weeks to reach $0.05 \mathrm{mg} . \mathrm{L}^{-1}$ and $0.1 \mathrm{mg} . \mathrm{L}^{-1}$ as final concentration. Pleurodeles $(P$. waltl) and Chironomids (C. riparius) were added subsequently during the fourth week while a second sampling of microbial consortium was performed at the end of that same week (T1). All organisms were sampled at the beginning of the $6^{\text {th }}$ week to perform analysis of the different endpoints (T2).

\subsection{Endpoint measurement}

\subsubsection{Primary compartment: sampling, DNA Extraction, PCR and sequencing}

Water and sediment were sampled at 3 different times during the experiment (T0, T1 and T2). At each sampling time, $100 \mathrm{~mL}$ of water and $10 \mathrm{~mL}$ of sediment were taken. Water samples were filtered on a $5 \mu \mathrm{m}$ filter (Whatman ${ }^{\circledR}$ Nuclepore $^{\mathrm{TM}}$ Track-Etched Membranes) in order to recover microorganisms from the water column. Filters and sediment samples were snap-frozen in liquid nitrogen and stored at $-80^{\circ} \mathrm{C}$ before processing for analyses. Total DNA was extracted from water samples and sediment subsamples $(644.6 \pm 37.2 \mathrm{mg})$ using the Mobio® power soil DNA isolation kit following manufacturer's instructions. Additionally, negative extraction controls were performed to ensure the absence of DNA contamination during the process. The 
quantity and quality of DNA extracts was analyzed using a NanoDrop 2000 UV spectrophotometer (Thermo Scientific).

The V4-V5 region of archeae and bacteria 16S rRNA gene was targeted using PCR1_515F (5'GTGYCAGCMGCCGCGGTA-3') and PCR1_928R (5'-CCCCGYCAATTCMTTTRAGT-3') primers set [49]. PCRs were performed on a Gene-AmpTM PCR system 9700 thermocycler (Applied Biosystems, Forster City, CA, United States) in a final volume of $50 \mu \mathrm{L}$ containing: $37.5 \mu \mathrm{L}$ of PCR water, $5 \mu \mathrm{L}$ of 10X PCR buffer, $2 \mu \mathrm{L}$ of extracted DNA, $2 \mu \mathrm{L}$ of both primer $(10 \mu \mathrm{M}), 1 \mu \mathrm{L}$ of dNTP $(2.5 \mathrm{mM})$ and $0.5 \mu \mathrm{L}$ of Taq DNA polymerase $(5 \mathrm{U} / \mu \mathrm{L}-$ Sigma Aldrich). The following PCR protocol was applied: $94^{\circ} \mathrm{C}$ for $120 \mathrm{~s}, 30$ cycles of $94^{\circ} \mathrm{C}$ for $60 \mathrm{~s}$, $65^{\circ} \mathrm{C}$ for $40 \mathrm{~s}, 72^{\circ} \mathrm{C} 30 \mathrm{sec}$ and $72^{\circ} \mathrm{C}$ for $10 \mathrm{~min}$.

Sequencing of amplicons from 16S rRNA genes was performed using Illumina MiSeq technology (2x250 pb) by the Get_PlaGe platform (Genotoul, Toulouse, France). Bioinformatic analysis was performed using FROGS (Find Rapidly Operational Taxonomic Units (OTU) Galaxy Solution) pipeline on Galaxy [50]. Briefly, sequences with mismatches in the primers were excluded and PCR primers were trimmed. Reads were clustered into OTUs using the Swarm clustering method [51]. Chimera were removed and filters were applied to keep OTUs present in at least 3 samples and representing at least $0.005 \%$ of all sequences [52]. 478 OTUs were assigned at different taxonomic levels (from Kingdom to species) using RDP classifier and NCBI Blast+ on Silva 132 database (pintail 80) [53].

\subsubsection{Chironomids}

At the end of the experiment, mortality, growth and teratogenicity were assessed in chironomid larvae that were not predated by Pleurodeles. A careful sorting of the sediment allowed to count the surviving individuals which were then measured using the image-J software (total length and width of the cephalic capsule). The cephalic capsules were recovered and discolored in potassium hydroxide $\left(15 \%, 95^{\circ} \mathrm{C}, 15 \mathrm{~min}\right)$, then mounted on microscope slides with quickhardening medium (Sigma). Teratogenecity was assessed by counting the number of deformities in mentum. According to Salmelin et al. [38], only absolute deformities such as missing and extra teeth and Köhn gaps were taken into account.

\subsubsection{Leaf litter degradation}

Alder leaves degradation was estimated by the total surface of degradation after microcosm exposure. Leaves were scanned at the beginning and at the end of experiment using a flatbed 
scanner with 600 DPI resolution. Leaves pictures were processed with image-J software: a 8 bit transformation and thresholding allowed us to quantify the degraded surfaces.

\subsubsection{Amphibians}

No endpoint was measured in $X$. laevis tadpoles that were all predated during the experiment by Pleurodeles larvae. For Pleurodeles larvae, mortality, growth and genotoxicity were studied after 10 days of exposure ( $\mathrm{n}=15$ for each endpoint per microcosm). Mortality was checked daily throughout the exposure. Growth was studied by measurements of size (photo and image analysis with Image-J software) at the beginning and end of the experiment. Genotoxicity was assessed by enumeration of micronucleated erythrocytes (MNE) in blood smears after fixation (methanol 99.9\%) and staining (groat hematoxylin). The number of cells containing one or more micronuclei was counted among a total of 1000 cells.

\subsection{Statistical analysis}

Statistical analysis was performed using Minitab 16 Statistical software. Data of growth $(P$. walt and C. riparius), development and survival chironomids (growth, development and survival) as well as leaf litter degradation endpoints were analyzed using one-way ANOVA when assumptions of normality and homogeneity of variance were met. As it was not the case for genotoxicity data, non-parametric Kruskal-Wallis followed by Dunn's multiple comparison test was performed. For metagenomics analysis, data manipulations, OTUs counts, alpha diversity indexes and Unifrac Distances calculations as well as multidimensional scaling (MDS) plot were carried out using "Phyloseq" R package [54]. Differential abundance of bacterial genera between exposed conditions compared to the control group was examined using "Deseq2" R package [55]. PERMANOVA was performed using Adonis function from the "vegan" R package [56]. The relationships between the biological responses measured were explored by multivariate Principal Component Analysis (PCA) and correlation analyses using “ade4 R" package [57].

\section{Results}

\subsection{Behavior of GO in exposure media}

The variation of the transmission data (compared to t0) vs time for EDI and Volvic are presented in Figure 2. While the dispersion is stable over 24 hours in EDI medium, a clear homogeneous sedimentation is evidenced in the case of the Volvic water after 5 hours (Figure S1). 


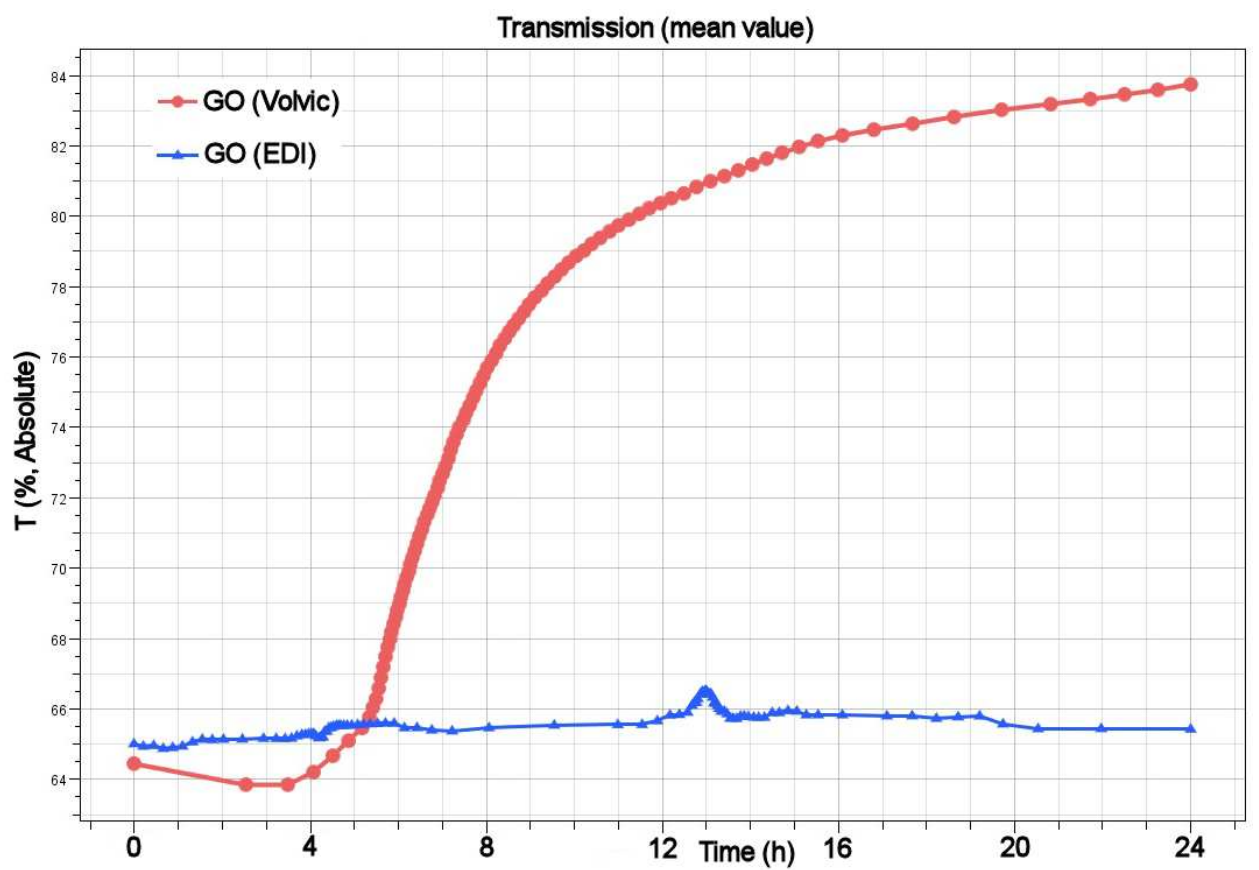

Figure 2: Variation of the transmission vs time for suspensions of GO at $10 \mathrm{mg} . \mathrm{L}^{-1}$ in deionised water (blue) or Volvic water (red).

\subsection{Effects on lower trophic level}

Bacterial communities in water compartment were different from those in sediment compartment regardless of time and GO concentration, as revealed by MDS and PERMANOVA analysis using unweighted UniFrac distances (Figure S2) $\left(\mathrm{F}=32.554 ; \mathrm{r}^{2}=\right.$ $0.385 ; p=0.001)$. Thus, the two compartments were studied separately to analyse the effects occurring on compartment associated bacterial communities. At T0, prior to contamination, community structures in the water were similar between the condition-related microcosms (PERMANOVA: $\left.\mathrm{F}=1.193 ; \mathrm{r}^{2}=0.284 ; \mathrm{p}=0.132\right)$, and the same was observed in sediments $(\mathrm{F}$ $=1.143 ; \mathrm{r}^{2}=0.276 ; \mathrm{p}=0.252$ ).

\subsubsection{Effects on microorganisms from the water column}

When analysing the alpha-diversity, no difference was measured for the observed diversity, Chao1 and Shannon indexes in the water column, during the whole experiment (Figure S3A). However, exposure duration as well as GO concentration were shown to significantly affect the microbial community structure (PERMANOVA: Contaminant: $\mathrm{F}=1.657 ; \mathrm{r}^{2}=0.104 ; p=0.010$; Exposure duration: $\left.\mathrm{F}=3.428 ; \mathrm{r}^{2}=0.216 ; p=0.001\right)$. MDS analysis indicated a higher contribution of the exposure duration (first axis) toward bacterial community composition compared to GO concentration (second axis) (Figure S3B). In the water column at T0, over 
$95 \%$ of overall community was represented by phyla Proteobacteria and Bacteroidetes with a relative abundance of $58.8 \pm 21.6 \%$ and $37.5 \pm 22.2 \%$ respectively (Figure $2 \mathrm{~A}$ ). The relative abundance of none of the phyla analysed was shown to be impacted by GO contamination (Table S2). Focusing on families from the two main phyla found in the water column (Figure S4), almost no effects were observed on relative abundances (Table S2). Analysis of the genera differential abundance in the GO conditions compared to the control group during the overall exposure led to a significant decrease of 3 genera from the phylum Proteobacteria (Legionella, Sphingobium and Reyranella) when exposed to $0.05 \mathrm{mg} . \mathrm{L}^{-1}$ of GO, while three other genera from this phylum were decreased in the $0.1 \mathrm{mg} . \mathrm{L}^{-1}$ exposure condition (Pseudominobacter, Sphingorhabdus and Devosia) (Figure S5A).

\subsubsection{Effects on microorganisms in sediment}

A significant increase in the biofilm observed richness and Chao1 was noticed between $\mathrm{T} 0$ and $\mathrm{T} 1$ without being influenced by GO concentration and remained stable until T2 while values of the Shannon index were not significantly influenced (Figure 4A). Exposure duration and GO concentration significantly affected microbial community structure (PERMANOVA: Contaminant: $\mathrm{F}=2.862 ; \mathrm{r}^{2}=0.161 ; p=0.002$, Exposure duration: $\mathrm{F}=4.875 ; \mathrm{r}^{2}=0.274 ; p=$ 0.001 ) with a greater contribution of the exposure duration over the contaminant concentration (Figure 4B). At T0, prior to contamination by GO, three main phyla constituted the bacterial compartment of the biofilm. Phyla Proteobacteria, Bacteroidetes and Planctomycetes relative abundances were of $29.1 \pm 15.1 \%, 10.6 \pm 4.2 \%$ and $3.8 \pm 2 \%$ respectively. The chloroplastic 16S rRNA gene from diatoms was affiliated to Ochrophyta phylum. This phylum represented $48.7 \pm 20.4 \%$ of the overall biofilm organisms (Figure $2 \mathrm{~B}$ ). The relative abundance of the two main bacterial phyla and diatoms was not affected by exposure to GO, while the phyla Planctomycetes, Armatimonadetes and WPS-2 significantly increased in the presence of GO at $0.1 \mathrm{mg} . \mathrm{L}^{-1}$ compared to the control group (Table S3). The effects occurring at the family scale in the three main bacterial phyla from the sediment were analysed (see supplementary data and Figure S6). Analysis of the OTUs differential abundances in the biofilm indicated that among 517 OTUs, exposure to $\mathrm{GO}$ at $0.05 \mathrm{mg} . \mathrm{L}^{-1}$ led to significant changes in the relative abundance of 15 taxa, among which 5 were found in a higher abundance and 10 in a lower abundance (Figure S5B). At $0.1 \mathrm{mg} . \mathrm{L}^{-1}, 20$ taxa were differentially abundant compared to the control with an equal distribution between over and under represented taxa. The relative abundance of genera Gemmata, Azospirillum and Flavobacterium were shown to decrease in a similar manner 
305 Armatimonas increased in the same order of manitude.

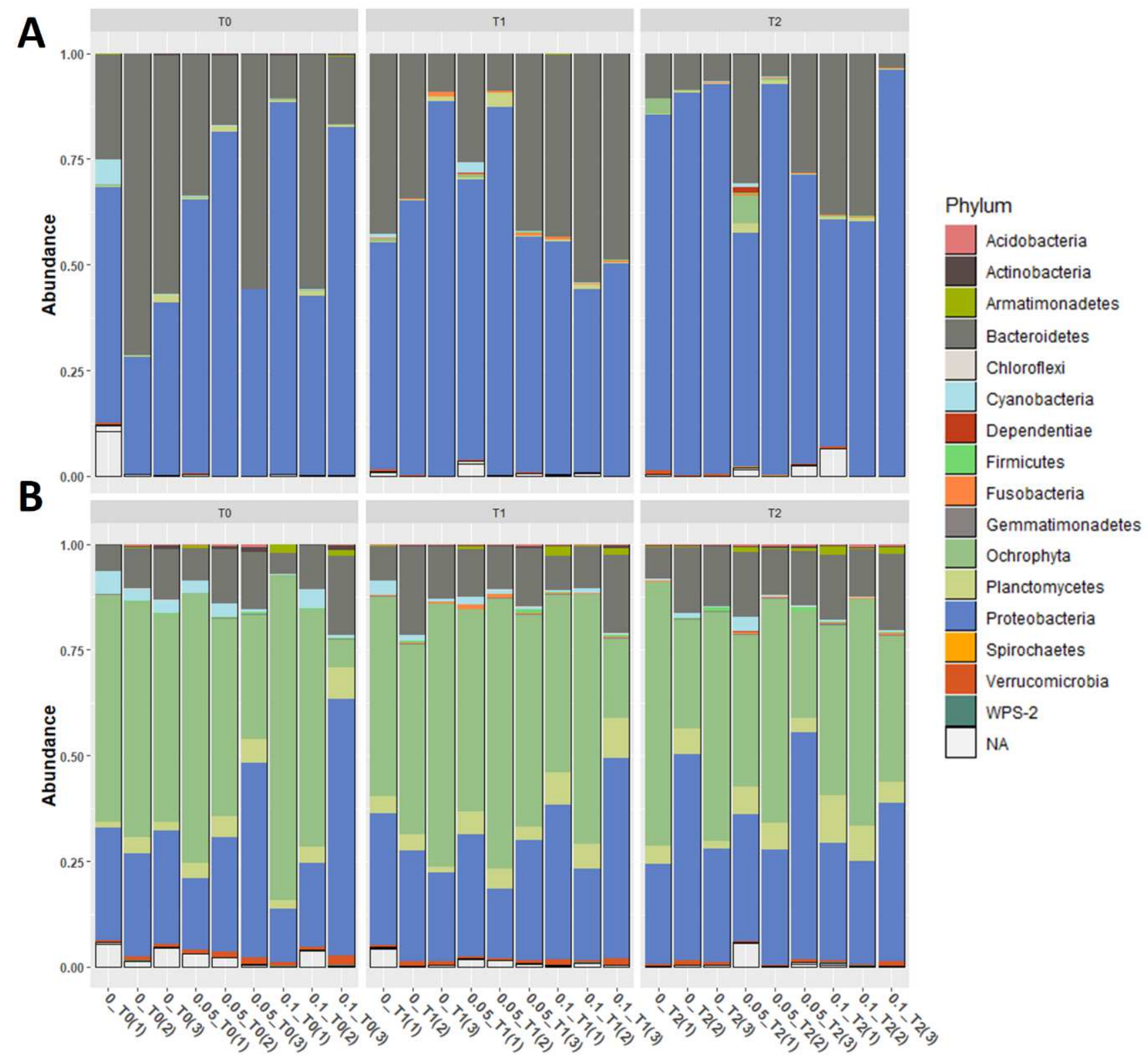

307 Figure 2: Relative abundance of bacterial phyla from the water column (A) and in the biofilm of the sediment (B) in function of GO concentration (0, 0.05 and $\left.0.1 \mathrm{mg} . \mathrm{L}^{-1}\right)$. 

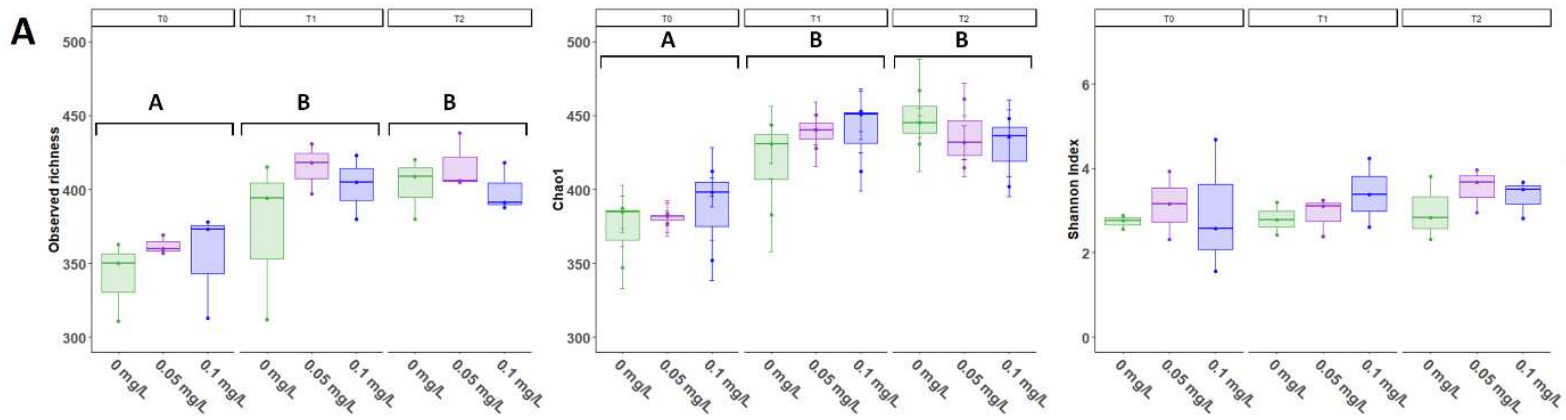

B

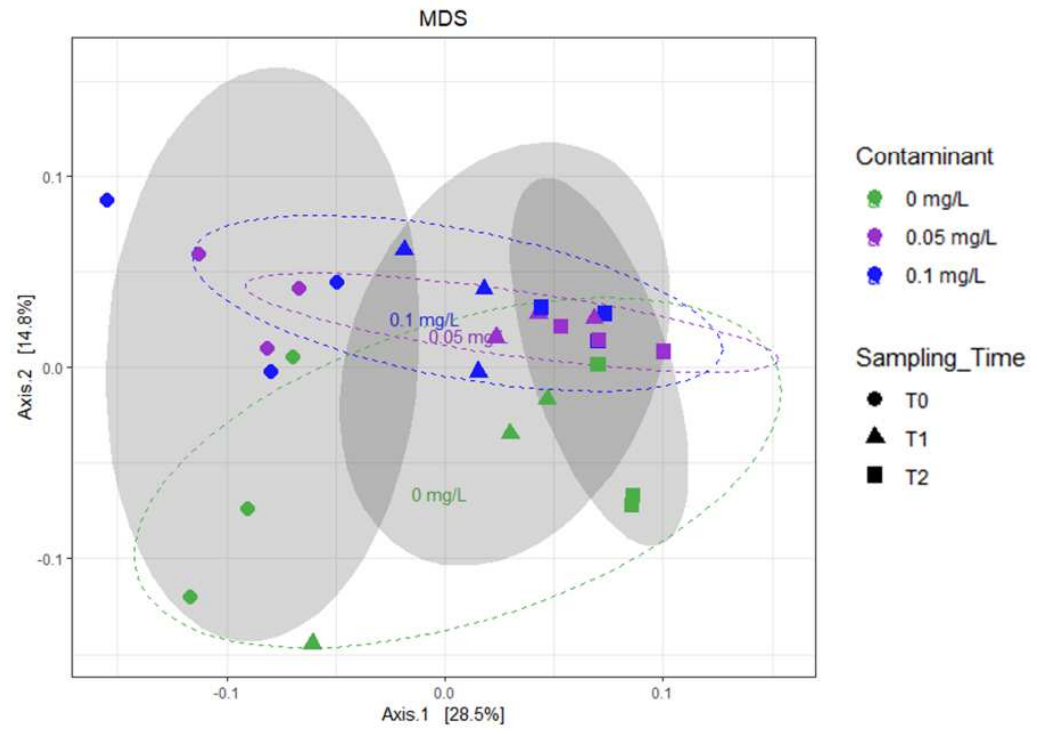

Figure 4: Effects of exposure to GO on microbial communities in the sediment of the microcosms, as revealed by $16 \mathrm{~S}$ amplicon sequencing. Observed richness, Chao1 and Shannon evenness indexes are compared between the three exposure conditions $\left(0 \mathrm{mg} . \mathrm{L}^{-1}, 0.05 \mathrm{mg} . \mathrm{L}^{-1}\right.$ and $0.1 \mathrm{mg} . \mathrm{L}^{-1}$ ) (A). MDS plot of bacterial communities based on unweighted Unifrac distances (B). Samples from different GO concentration are represented by different colors with $95 \%$ confidence ellipses, while different sampling times are represented by forms surrounded by 95\% confidence ellipses in grey color.

\subsection{Effects on chironomids}

At the end of the experiment, no significant difference was noticed on the survival rate of $C$. riparius whatever the exposure condition (ANOVA, $\mathrm{p}=0.976$ ). A mean of $8.02 \pm 4.7 \%$ of the overall chironomids were remaining for all conditions. Larval growth and determination of development stage measured from length of the organisms and head width measurement respectively, were not significantly affected by GO exposure (ANOVA, p $=0.280$ and 0.860 respectively). Considering the whole organisms, $60.6 \%$ of the larvae reached the stage 4 at the end of the experiment and a mean size of $8.6 \pm 2.1 \mathrm{~mm}$ was measured. Analysis of teratogenicity in stage 4 larvae indicated no effect of GO exposure (ANOVA, p = 0.913). 
327 At the end of the exposure, a significant increase in leaf degradation was noticed after exposure to $0.05 \mathrm{mg} . \mathrm{L}^{-1}$ of $\mathrm{GO}$ compared to the control group while only a trend in increase of degradation was observed at $0.1 \mathrm{mg} . \mathrm{L}^{-1}$ (ANOVA $p=0.027$ followed by Tuckey) (Figure 5).

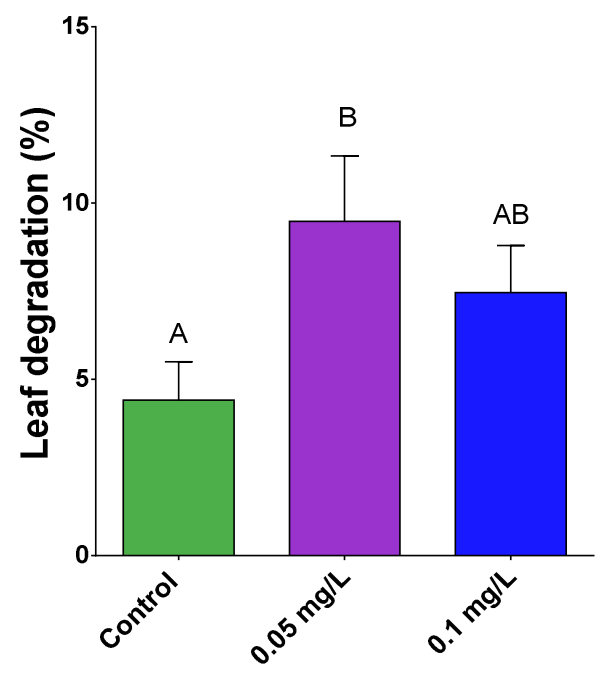

331 Figure 5: Alder leaves degradation measured at the end of the exposure to 0, 0.05 or $0.1 \mathrm{mg} . \mathrm{L}^{-}$ 3321 of GO. Data are presented as mean \pm SEM $(n=39$ leaves per condition). ANOVA $p<0.05$ followed by Tukey test. Letters indicate significant differences between concentrations tested.

\subsection{Effects on Pleurodeles larvae}

No mortality in Pleurodeles larvae was observed during the whole experiment and larval growth was not affected by GO exposure (ANOVA, $p=0.723$ ). At the end of the experiment, a significant increase in micronucleated erythrocytes was observed in larvae exposed to $0.1 \mathrm{mg}$. $\mathrm{L}^{-}$

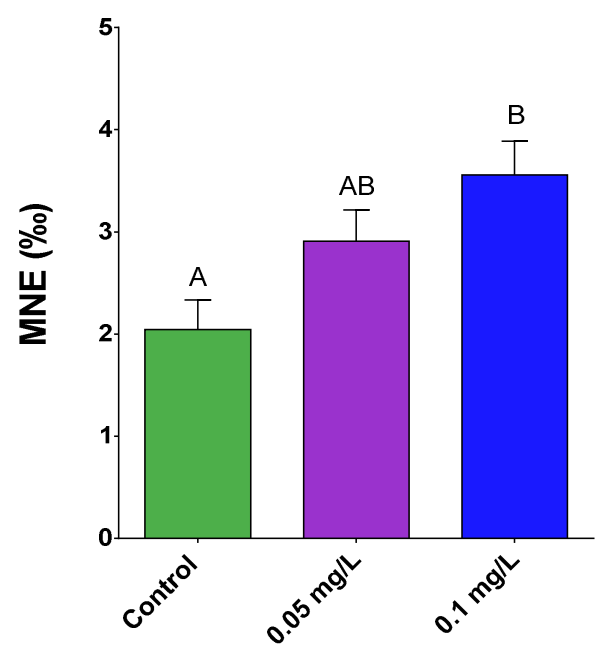


Figure 6: Micronucleus induction measured in erythrocytes of Pleurodeles waltl exposed using microcosm to GO at T2 (10 days of exposure). Data are presented as mean $\pm \mathrm{SD}(\mathrm{n}=45$ larvae per condition). Kruskal-Wallis $p<0.01$ followed by multiple rank comparisons. Letters indicate significant differences between concentrations tested.

\subsection{Principal Component Analysis}

345 In the principal component analysis of the responses measured at the different trophic levels, 346 PC1 and PC2 explained 38,6\% and $24.3 \%$ of the total variance respectively (Figure 7). The PCA showed a better segregation between the controls microcosms and those contaminated with GO along the PC2 axis based on biological responses. The parameters related to chironomids development and survival as well as microbial diversity were negatively associated with PC1 while teratogenesis was positively associated with PC1. The leaf litter degradation and genotoxicity in pleurodeles were mainly associated with the conditions of GO exposure along PC2.

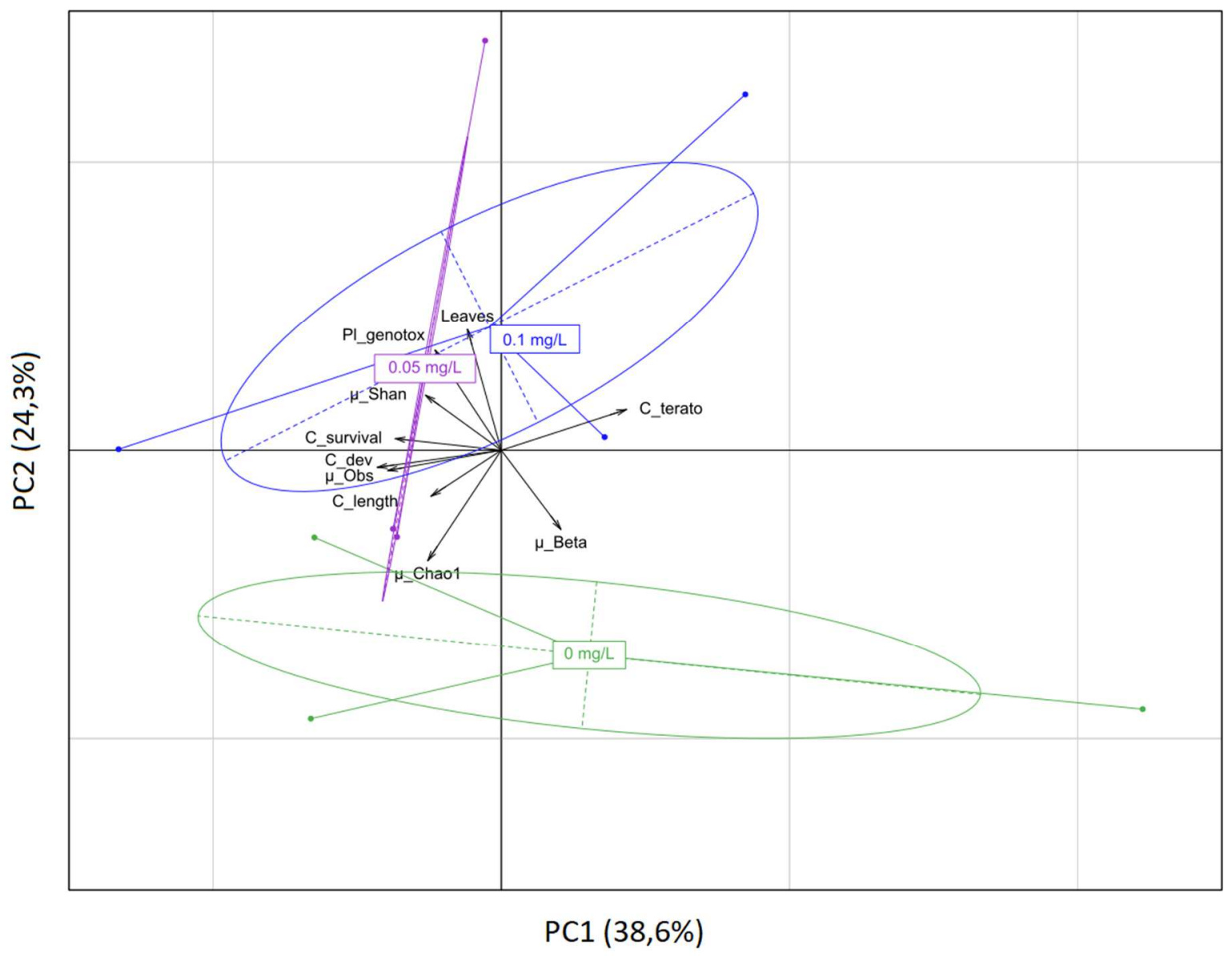

Figure 7: Biplot of the first two components of principal component analysis (PCA) including biological responses measured in different conditions of GO exposure $\left(0,0.05\right.$ or $\left.0.1 \mathrm{mg} . \mathrm{L}^{-1}\right)$. 
Axis 1: $38.6 \%$, Axis $2: 24,3 \% . \mathrm{Pl}=$ pleurodeles, $\mathrm{C}=$ Chironomus, dev = development stage, terato $=$ teratogenesis $\mu=$ microorganisms, Obs $=$ Observed diversity, Shan $=$ Shannon index, Beta $=$ beta diversity, Leaves $=$ leaf litter degradation.

\section{Discussion}

To our knowledge, this study is the first one to investigate the effect of GO under environmentally relevant conditions using a reconstituted trophic chain through the use of microcosms. Because of the limited available techniques to quantify GO in complex matrix and changes of materials characteristics over time at environmentally realistic concentrations [58], no relevant quantification analysis could be performed in our study. Thus, the hypothesis of material distribution in our system and toxicological mechanisms involved are based only on Turbiscan data and biological responses measured.

\subsection{Behavior of GO in exposure media}

The fate, transport, and bioavailability of GO are mainly determined by their behaviour in aquatic ecosystems including aggregation deposition that can be influenced by multiple factors [66]. On the one hand, interactions with organic matter of the experimental system would increase the colloidal stability of the GO $[11,67,68]$, while on the other hand, the presence of ions in the water such as $\mathrm{Ca}^{2+}$ or $\mathrm{Na}^{+}$could adsorb on the negatively charged functional groups of GO, leading to reduced surface charge and reduced dispersion stability, leading to its deposition [69]. According to the obtained results it seems that the interactions with ions of the exposure media occurring under the experimental conditions are responsible for the sedimentation of the GO. Such sedimentation of the material would decrease its bioavailability for organisms from the water column while it will increase for organisms living at the sedimentwater interface.

\subsection{Effects on microorganisms}

Studying the effects of GO on microorganisms is essential as they constitute the first link of the food chain. Literature is furnished of data demonstrating the antibacterial capacities of GO [2426,59-61]. However, few studies focused on the effects generated by CBNs on complex bacterial communities from aquatic ecosystems and the data available were mainly obtained on bacterial consortium from soils [62,63] or activated sludge [64,65]. In the present study, complex changes were observed in the bacterial compartment over the whole experiment. It is interesting to point out that GO exposure induced only minor differences in bacterial 
communities in the water column whereas the biofilm communities were more impacted. The difference in magnitude of GO impact between water and sediment communities associated to Turbiscan analysis confirm that the GO is more bioavailable for microbial communities from the sediment rather than for free living microorganisms from the water column. The presence of a complex biofilm containing diatoms N.palea and bacteria also contribute to this change of bioavailability. Indeed, these microorganisms were shown to stabilize CBNs in the biofilm through secretion of extracellular polymeric substances (EPS) [70-72]. Thus, the trapping of GO may decrease its bioavailability for bacteria from the water column while it would increase the occurrence of direct contact with bacteria in the biofilm (i.e. and other benthic organisms). This direct contact was evidenced as the main mechanism of cytoxicity toward bacteria through impairment of cell membrane integrity [27,73,74].

Our data show that the biofilm compartment is more dynamic compared to free living bacteria as indicated by the time effect on diversity indexes from the control group. However, as there are no other organisms in the microcosms at T0, introduction of chironomids and newts between $\mathrm{T} 0$ and $\mathrm{T} 1$ would contribute mainly to the biofilm's dynamics through the increase in bacterial alpha diversity via the production of faeces, for example. In spite of this temporal dynamics inherent to microcosm experimental design as well as to this form of bacterial life [75], beta diversity measurement in pelagic as well as in benthic communities evidenced diverging phylogenetic trajectories in presence of GO compared to the control group. In this case, the stronger the contamination pressure is, the more communities are diverging. As the multiple species in biofilms may possess different sensitivity to GO, the decrease of affected taxa make available new ecological niches benefiting to less sensitive and more opportunistic species leading to different equilibrium in microbial communities. Thus, the phyla Planctomycetes, Armatimonadetes and WPS-2 appear tolerant to GO as their relative abundances increased in the exposed conditions. A similar trend was previously evidenced for Planctomycetes from soil bacterial communities exposed to GO [76] or silver nanoparticles [77]. These changes would contribute to the divergences in the evolution of the biofilm composition between the tested conditions. However, as a dynamic system, it is possible that the direct toxicity of GO will be attenuated with biofilm maturation. Indeed, it was previously observed in bacterial biofilm using E. coli or $S$. aureus models that after 48 hours of exposure, corresponding to the mature phase of biofilm, cell growth inhibition and induction of ROS production by rGO was suppressed, even at concentrations up to $100 \mathrm{mg} . \mathrm{L}^{-1}$ [78]. This study also indicated that bacteria were able to oxidize rGO into GO. Transformations of the tested material potentially occurring 
under our experimental conditions are remaining to be assessed when technological barriers will be overcome.

\subsection{Effects on chironomid larvae and consequences for leaf litter degradation}

No apparent toxicity on growth and teratogenicity could be highlighted on chironomids. The low amount of larvae remaining at the end of the exposure could be only related to predation by Pleurodeles larvae that is more likely to occur within the last days of the experiment when they reach larger sizes [43,79]. It is also possible that the apparent no effects on chironomids may result from a preferential predation by pleurodeles larvae of affected chironomids. However, the low sensitivity of chironomids toward CBNs was previously indicated for fullerene [80], multi-walled carbon nanotubes [81] as well as GO [67]. In the later study, no effects were observed on chironomids after 9 days of exposure to $\mathrm{GO}$ at concentrations up to $100 \mathrm{mg} . \mathrm{L}^{-1}$, which is consistent with the present results and corroborate the predation hypothesis without any pressure of selection.

Chironomids are shredders, deposit feeders that preferentially feed on biofilm rather than nonliving organic matter [82]. Despite the known implication of bacteria in this ecological process, chironomids constitute the main decomposers of organic matter in such time-duration experiment [22]. Despite the pressure of predation on chironomids, the alder leaves decomposition process is maintained. In this study, it appears that this process increases in presence of GO while opposite results were observed in the case of contamination by other nanoparticles $[22,83]$. It was previously observed through the comparison of deformities in the mouthparts of chironomids sampled in differentially contaminated sites that teratogenicity was associated with lower leaves degradation activities [84]. In the present study, no teratogenicity was measured and as PCA results indicated an independence of these variables, we can suggest that the increase of leaves consumption is associated to indirect effects. The presence of GO in the biofilm might influence their feeding behaviour through two possible ways. 1) Changes in bacterial communities and GO trapping might decrease the biofilm palatability. Indeed, it was demonstrated that food quality prevails on contaminant concentration for chironomids food source selection [85]. Thus, the loss of biofilm palatability and quality would lead to preferential feeding on alder leaves. 2) The presence of GO at low concentration may increase fungus development as it was demonstrated that GBMs could stimulate the growth of rot fungus at "low concentration" without influencing the decomposition activity [86,87]. Thus, an increase in fungus biomass on leaves would improve their palatability and influence chironomids feeding behaviour $[88,89]$. According to the PCA results, it seems that there is a correlation 
between the diversity of microorganism species from the biofilm and the development of chironomids that is consistent with the first hypothesis. It is interesting to note that in any case, this change of behaviour had no measurable consequence on chironomids development. Modifications of the feeding behaviour could also theoretically contribute to changes observed in bacterial community compositions. Indeed, diet changes could lead to modification of chironomids gut microbiota and modify microbial composition of the faeces rejected into the mesocosms.

These changes in organic matter decomposition could potentially lead to changes in carbon and nutrient cycling, influencing energy flows of the system [90] but it remains to be determined whether or not it is occurring under these conditions. Thus, litter decomposition appears as an interesting marker integrating direct and indirect toxicological effects occurring at the lower trophic levels.

\subsection{Effects on pleurodeles larvae}

The results obtained in this experiment indicated that GO is able to induce genotoxic effects in vivo at low concentration under realistic environmental conditions. Genotoxic potential of GO was also observed in other organisms such as mice after repeated or single injection of GO at concentrations ranging from 0.01 to $20 \mathrm{mg} \cdot \mathrm{kg}^{-1}$ [91,92]. This was also observed in the amphibians Xenopus laevis [42], which is consistent with the present results. In the latter study case, tadpoles were exposed during 12 days to the same commercial GO as the one used in the current microcosm study. The genotoxic response measured in $X$. laevis after exposure to 0.1 mg. $\mathrm{L}^{-1}$ was associated to oxidative stress and pro-inflammatory response leading to an increase in circulating micronucleated erythrocytes [42]. Despite important biological differences between these two amphibian model species, it can be suggested that the genotoxic effects observed in Pleurodeles resulted from similar pathways. Under our experimental conditions, Pleurodeles larvae were exposed to GO through two pathways simultaneously: direct exposure due to the presence of GO in the media as well as dietary exposure by feeding on contaminated chironomids. However, PCA analysis indicated that the marker of genotoxicity was independent from those associated to other trophic levels, which reinforces the hypothesis of effects associated to direct exposure over trophic effects. In the case of a direct exposure, as the contamination was performed sequentially and according to previous study indicating that at least 4 days of exposure to a genotoxic compound are necessary to induce the formation of micronuclei [47], we can suggest that a concentration of $0.8 \mathrm{mg} \cdot \mathrm{L}^{-1}$ of $\mathrm{GO}$ is able to induce genotoxic effects (concentration reached 4 days before the sampling). It seems that the 
Table 1 Characteristics of the patients, physicians and pharmacists

\section{Patients}

$\mathrm{n}=768$

$507(66 \%)$

[18-39]: 193 (25\%)

[40-54]: 215 (28\%)

[55-64]: $141(18 \%)$

[65-74]: 127 (17\%)

[75-84]: $74(10 \%)$

[85 and more]: $17(2 \%)$

Data missing: $1(0.1 \%)$
Physicians

$\mathrm{n}=345$

$145(42 \%)$

Mean $49.7 \pm 11.0$ years

Median 51 years [Min: 27, Max: 69]
Pharmacists

$\mathrm{n}=198$
Female

Age

\section{Gender $n(\%)$}

\section{Environment n (\%)}

\section{Urban}

Semi-urban

Rural
$206(26,9 \%)$

$188(24,5 \%)$

$372(48,6 \%)$
$118(34 \%)$

$123(36 \%)$

$104(30 \%)$
$127(64 \%)$

Mean 45,3 $\pm 5,7$ years

Median 46 years [Min: 24, Max: 71]
$67(34 \%)$

48 (24\%)

$83(42 \%)$ 


\section{Data missing}

Mode of working (physicians) (\%)

Associate $242(70 \%)$

Alone 103 (30\%)

Number of pharmacists in the pharmacy, $n(\%)$

1: $18(9 \%)$

2: $98(50 \%)$

3: $63(32 \%)$

4: $18(9 \%)$

Data missing: $1(0.5 \%)$

Number of different medications taken daily

Mean 1,89 $\pm 2,22$ medications/day

Median 1/day [min: 0, max:10]

Number of patients according to the number of

different medications taken daily; $n,(\%)$

0 or 1 medications/day

$429(55.8 \%)$

2 to 4 medications/day

$235(30.6 \%)$ 


\section{5 or more medications/day}

\section{Data missing}

Number of patients reporting having already

confused two tablets or capsules n (\%)
$98(12.8 \%)$

$6(0.8 \%)$

$77(10 \%)$ 